Review

\title{
Key cell signaling pathways modulated by zerumbone: Role in the prevention and treatment of cancer
}

\author{
Remya Prasannan ${ }^{\text {a }}$, Karunakaran A. Kalesh ${ }^{\text {b }}$, Muthu K. Shanmugam ${ }^{\text {a }}$, Alamelu Nachiyappan ${ }^{\text {a }}$ \\ Lalitha Ramachandran $^{a}$, An H. Nguyen ${ }^{a}$, Alan Prem Kumar a,c,d,e, Manikandan Lakshmanan ${ }^{f}$, \\ Kwang Seok Ahn ${ }^{\text {g,**, Gautam Sethi }}{ }^{\text {a,d,* }}$ \\ ${ }^{a}$ Department of Pharmacology, Yong Loo Lin School of Medicine, National University of Singapore, Singapore 117597, Singapore \\ ${ }^{\mathrm{b}}$ Department of Chemistry, National University of Singapore, Singapore 119077, Singapore \\ ' School of Biomedical Sciences, Faculty of Health Sciences, Curtin University, Western Australia 6009, Australia \\ ${ }^{\mathrm{d}}$ Cancer Science Institute of Singapore, National University of Singapore, 28 Medical Drive, Singapore 117456, Singapore \\ e Department of Biological Sciences, University of North Texas, Denton, Texas, USA \\ ${ }^{\mathrm{f}}$ Department of Pharmaceutical Technology, Jadavpur University, Kolkata 700032, India \\ ${ }^{\mathrm{g}}$ College of Oriental Medicine, Kyung Hee University, Seoul 130-701, Republic of Korea
}

\section{A R T I C L E I N F O}

\section{Article history:}

Received 8 June 2012

Accepted 16 July 2012

Available online 27 July 2012

\section{Keywords:}

Apoptosis

Cancer

NF- $\mathrm{B}$

TRAIL

Zerumbone

\begin{abstract}
A B S T R A C T
Phytochemicals and their synthetic derivatives are making a significant contribution in modern drug discovery programs by targeting several human diseases, including cancer. Most of these natural compounds are often multitargeted in nature, which is generally a very desirable property for cancer therapy, as carcinomas typically involve dysregulation of multiple genes and associated cell-signaling pathways at various stages of initiation, progression and metastasis. Additionally, these natural agents generally have lower side-effects, are readily available and hence are cost effective. One such natural compound is zerumbone, a cyclic eleven-membered sesquiterpene, isolated from the tropical plant Zingiber zerumbet Smith that has attracted great attention recently for its potent anticancer activities in several tumor models. This review summarizes the data based on various in vitro and in vivo studies related to the effects of zerumbone on numerous pivotal molecular targets in cancer and its reported chemopreventive/therapeutic effects in different models of cancer.
\end{abstract}

(c) 2012 Elsevier Inc. All rights reserved.

\section{Contents}

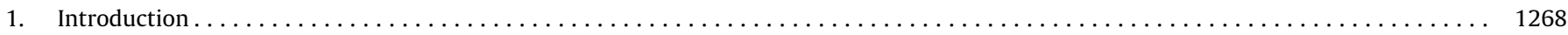

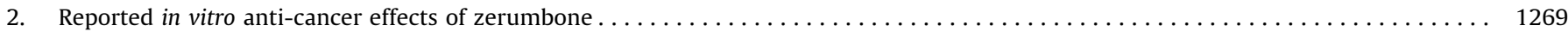

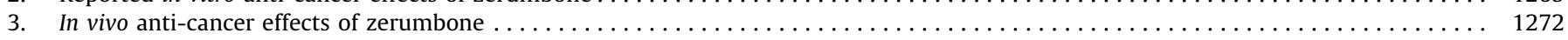

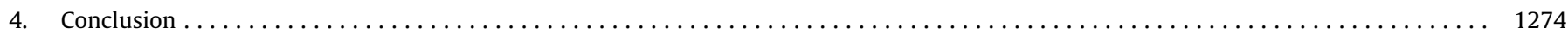

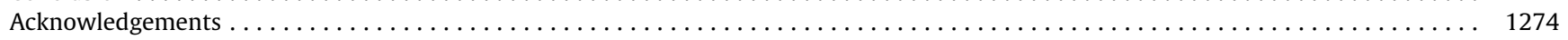

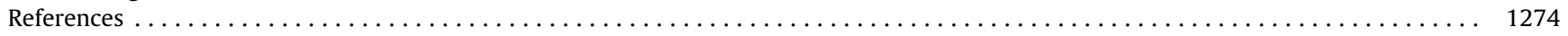

\section{Introduction}

Plant-derived natural products or 'phytochemicals' have been used for medicinal purposes for millennia [1-4]. Many ancient

\footnotetext{
* Corresponding author at: Department of Pharmacology, Yong Loo Lin School of Medicine, National University of Singapore, Singapore 117597, Singapore. Tel.: +65 65163267; fax: +65 68737690.

** Corresponding author. Tel.: +82 29612316.

E-mail addresses: ksahn@khu.ac.kr (K.S. Ahn), phcgs@nus.edu.sg (G. Sethi).
}

civilizations used edible plants, parts of plants and plant extracts for treating several human ailments [5-7]. With developments in modern science and technology, isolation of bioactive components from natural sources and identification of their molecular mechanism of action in the living system became an important pharmacological research [8-10]. Several promising natural products and natural product-inspired compounds are currently in clinical and pre-clinical developmental stages for cancer treatment [7,11-13]. Limited supply of active compounds from natural sources, difficulties in complete profiling of their cellular targets and characterization of their molecular mechanisms of 
action are among the key challenges in the natural product-based drug discovery programs [4,14]. Developments of ingenious approaches such as metabolic engineering and synthetic biology [6,15-17] are expected to provide solutions to maximize the production of important bio-active components. However, there is still an urgent need to accelerate the target profiling of various isolated natural compounds.

Interestingly, phytochemicals exhibit their anti-cancer properties via a variety of distinct mechanisms [18-20]. For instance, some act as cancer blocking agents, which essentially facilitate the detoxification of specific pro-carcinogens so that their activation to lethal carcinogens is prevented $[19,21]$. Others act as cancer suppressing agents, and can inhibit the development of cancerinitiated cells to pre-neoplastic and neoplastic cells [22,23]. However, in most cases, a given phytochemical can execute a multitude of intracellular effects, rather than a specific biological interaction, and the combination of these effects eventually facilitates cancer prevention/treatment [24,25]. Many phytochemicals are also found to modulate inflammation-related molecular targets [26,27]. Inflammation is a complex, multifunctional biological response of vascular tissues to tissue injury, characterized at the molecular level by the formation of a network of chemical signals that activate and direct migration of leukocytes from the venous system to the sites of injury $[19,28]$. While acute inflammation is an immediate response to injury, chronic inflammation leads to cellular environments with significant neoplastic risk $[21,29,30]$. Numerous cellular molecules serve as biomarkers of inflammation. These include pro-inflammatory cytokines such as tumor necrosis factor- $\alpha$ (TNF- $\alpha$ ), interleukin (IL) $-1 \alpha$, IL-1 $\beta$, chemokine CXC chemokine receptor 4 (CXCR4) and its ligand CXCL12, interferon- $\gamma$ (IFN- $\gamma$ ), inducible nitric oxide synthase (iNOS), adhesion molecules such as intracellular adhesion molecule 1 (ICAM-1), vascular cell adhesion molecule 1 (VCAM-1) and endothelial leukocyte adhesion molecule (ELAM) and angiogenic factors such as vascular endothelial growth factor (VEGF) [31-33]. As the functional and causal relationship of inflammation with several carcinomas becomes more evident in recent years, agents that are found to modulate inflammationrelated molecular targets are receiving substantial interest in anticancer drug development programs.

Among the various secondary metabolites (such as terpenoids, alkaloids, phenolic compounds, and glycosides) isolated from different plant sources, the terpenoids, obtained from the modification of basic five-carbon isoprene backbone units, form the largest class with more than 25,000 members identified so far $[34,35]$. According to the number of isoprene units in their backbone, the terpenoids are classified into hemiterpinoids (one isoprene unit, 5C), monoterpinoids (two isoprene units, 10C), sesquiterpinoids (three isoprene units, 15C), diterpinoids (four isoprene units, 20C), sesterterpinoid (five isoprene unit, 25C) and so on [36]. Many terpenoids show therapeutic potential as antibacterial [37], anti-viral [38], anti-malarial [39], anti-parasitic [40], anti-hyperglycemic [41], anti-inflammatory [42] and anti-cancer agents [36]. One such terpenoid compound that holds tremendous potential for the prevention/treatment of cancer is zerumbone (2,6,9,9-tetramethyl-[2E,6E,10E]-cycloundeca-2,6,10-trien-1-

one), a cyclic eleven-membered sesquiterpene, isolated from the rhizomes of the tropical plant Zingiber zerumbet Smith. Although the exact molecular mechanism(s) for the potential anti-cancer effects of zerumbone remains yet to be elucidated, existing literature suggests that it can modulate an array of important molecular targets both for the prevention and treatment of cancer (Fig. 1). In this minireview, we summarize the data from published literature on the reported in vitro and in vivo anti-cancer effects of zerumbone thereby, highlighting its potential therapeutic role in different cancers.

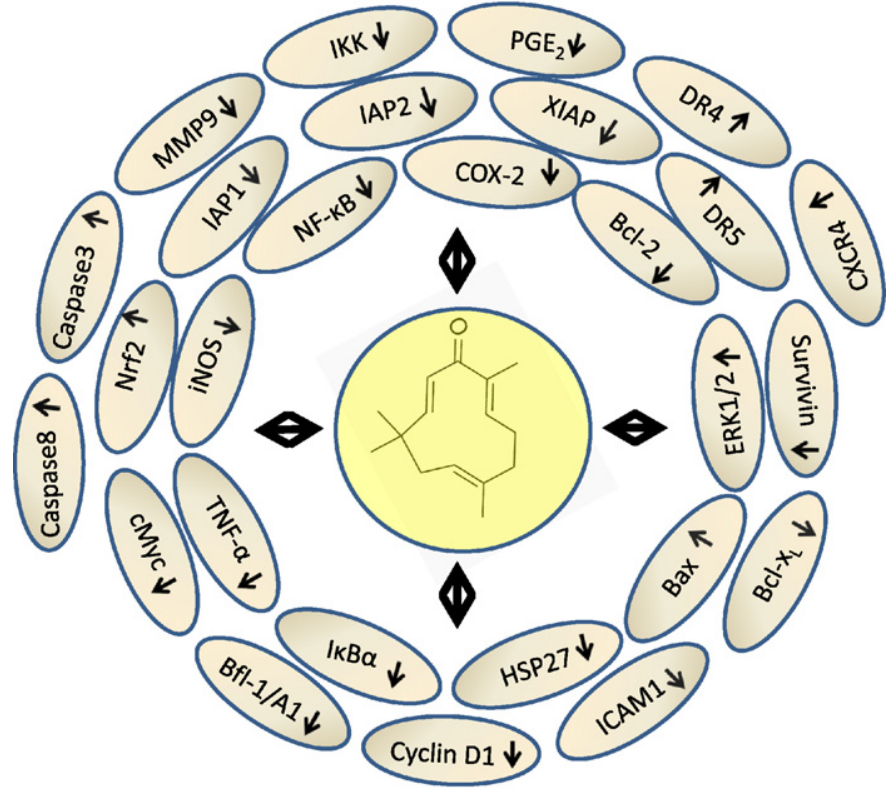

Fig. 1. Signal transduction and apoptotic pathways modulated by zerumbone in tumor cells and animal models. $\uparrow$ indicates increased expression/activation; $\downarrow$ indicates decreased expression/activation.

\section{Reported in vitro anti-cancer effects of zerumbone}

In vitro studies using a variety of cancer cell lines have so far provided important clues into the potential molecular targets of zerumbone. Murakami and co-workers reported concentrationdependent suppressive effect of zerumbone on 12-O-tetradecanoylphorbol-13-acetate (TPA)-induced superoxide anion radical $\left(\mathrm{O}_{2}{ }^{-}\right)$generation from both nicotinamide adenine dinucleotide phosphate oxidase in differentiated HL-60 cells and xanthine oxidase in AS52 CHO cells [43]. In both cases, complete abrogation of TPA-induced $\mathrm{O}_{2}{ }^{-}$generation was observed following zerumbone pre-treatment. On the other hand, $\alpha$-humulene, a structural analog of zerumbone lacking the carbonyl functional group, failed to generate any significant effect in $\mathrm{O}_{2}{ }^{-}$generation, pointing to the importance of the carbonyl functionality in zerumbone for its biological effects. Concentration-dependent suppression was also observed in combined lipopolysaccharide (LPS) and interferon gamma (IFN- $\gamma$ )-stimulated expression of iNOS and COX-2 proteins as well as in release of TNF- $\alpha$, both from RAW264.7 mouse macrophage cells. The treatment also resulted in decrease in concentration of nitrite and PGE2. Zerumbone treatment, however, was reported to produce no significant effect on the combined LPSand IFN- $\gamma$-stimulated expression of cyclooxygenase (COX)- 1 protein in the RAW264.7 cells. The researchers also observed dose-dependent inhibition of proliferation of human colonic adenocarcinoma cell lines LS174T, LS180, COLO205 and COLO320DM upon zerumbone treatment. Noticeably, zerumbone exerted far less anti-proliferative effects on normal human dermal cells (2F0-C25) and normal colon fibroblast cells (CDD-18 Co), indicating the selectivity of the compound toward cancer cells. Apoptosis induction by zerumbone was also observed in COLO205 cells. The results of Murakami and co-workers clearly highlight the potential of zerumbone in several suppressive activities in cancer cells. They also proposed the idea for the first time that many of these suppressive effects are possibly due to the effect of the compound on the nuclear factor-kappa B (NF- $\kappa B$ ) activity or on IkappaBalpha $(\mathrm{I} \kappa \mathrm{B} \alpha)$ kinase activity or both. However, in a subsequent paper, Murakami et al. reported that zerumbone at a tested dose of $20 \mu \mathrm{M}$ did not produce significant effect on the expression of NF- $\mathrm{KB}$ in RAW264.7 mouse macrophages [44]. 
Furthermore, they reported that zerumbone caused both LPSinduced mitogen activated protein kinases (MAPKs)/Akt activation and degradation of IкB $\alpha$ protein. The researchers also observed that zerumbone suppressed COX-2 mRNA induction. These results, taken together, made them to speculate the possibility of MAPK activated protein kinase-2 (MK-2), and molecules located further downstream of MK-2 in the COX-2 mRNA degradation pathway, as potential molecular target(s) of zerumbone.

Aggarwal and co-workers hypothesized that many of the observed in vitro activities of zerumbone (Table 1) could be explained by its ability to inhibit master transcription factor, NF$\kappa B$ [45]. Several genes regulated by NF- $\kappa B$ play a key role in proliferation, survival and metastasis of tumor cells [46-48]. Aggarwal and co-workers reported cell type-independent suppressive effect of zerumbone on the activation of NF- $\mathrm{KB}$ induced by stimuli such as cigarette smoke condensate, phorbol 12-myristate13-acetate, okadaic acid, TNF and hydrogen peroxide. The researchers also observed inhibition of constitutive NF- $\kappa B$ activation by zerumbone in FaDu and LICR-LON-NH5 tumor cells. Zerumbone also suppressed TNF-induced invasion activity of cancer cells, abolished TNF-induced І $\kappa \mathrm{B} \alpha$ protein degradation and inhibited TNF-induced phosphorylation of $\mathrm{I} \kappa \mathrm{B} \alpha$ protein and $\mathrm{p} 65$ protein. Interestingly, they also found that NF- $\kappa B$-regulated gene products involved in cell proliferation such as cyclin D1 and c-Myc; antiapoptosis such as survivin, inhibitor of apoptosis protein (IAP)1, (IAP)-2, X-linked inhibitor of apoptosis (XIAP), B-cell lymphoma 2 (Bcl-2), B-cell lymphoma-extra large $\left(\mathrm{Bcl}-\mathrm{x}_{\mathrm{L}}\right), \mathrm{Bcl}-2$-related protein (A1), and cellular FLICE-like inhibitory protein (c-FLIP); and invasion such as COX-2, matrix metalloproteinase-9 (MMP-9) and ICAM-1 were down-regulated upon zerumbone treatment.

Several lines of evidence point to the ability of zerumbone to induce apoptosis as the major cause for its anti-proliferative activity that has been observed in many tumor cell lines. Hawariah and co-workers reported anti-proliferative activity of zerumbone in human liver hepatocellular carcinoma (HepG2) cells in culture [49]. The researchers observed moderate selectivity in the antiproliferative activity of zerumbone for the liver cancer cells compared to non-malignant Chang liver cells and MDBK cells. Zerumbone was also reported to exert both improved cytotoxic effect and cancer cell selectivity compared to cisplatin, a widely used drug for the treatment of small cell lung cancer, ovarian cancer and several other carcinomas. Treatment of HepG2 cell lines with zerumbone at a fixed concentration of $3.45 \mu \mathrm{M}$ led to DNA fragmentation, time-dependent up-regulation of pro-apoptotic $\mathrm{Bcl}-2$-associated $\mathrm{X}$ protein (Bax) protein and time-dependent suppression of anti-apoptotic $\mathrm{Bcl}-2$ protein. The compound at the tested dose however, did not significantly affect expression level of the tumor suppressor $\mathrm{p} 53$ protein. The anti-proliferative activity of

Table 1

Selected in vitro anti-inflammatory and anti-cancer effects of zerumbone.

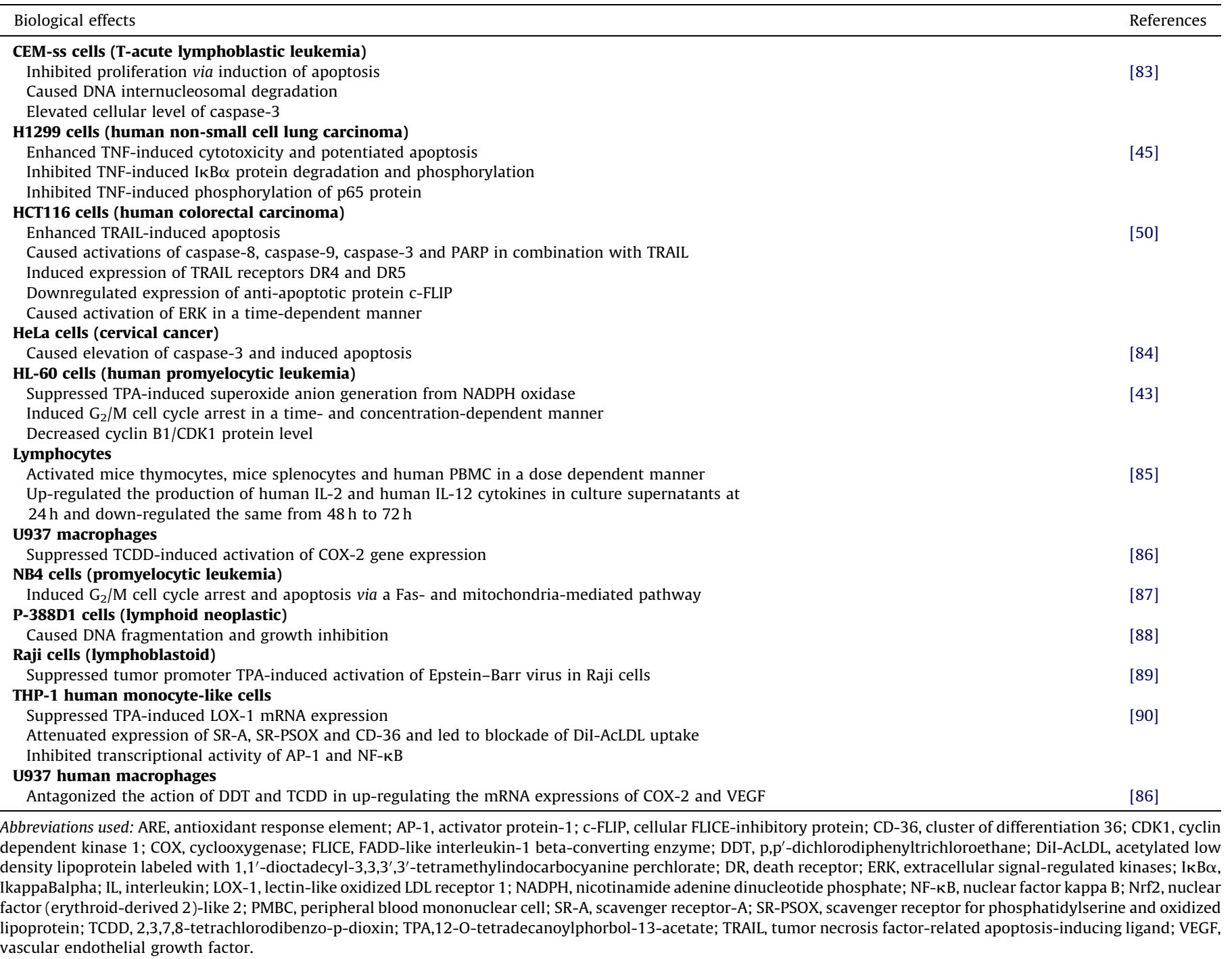


zerumbone in HepG2 cell line was, therefore, attributed to the potential role of the compound to act as an apoptosis inducer via modulating the Bax vs. Bcl-2 ratio without affecting the p53 expression. However, it remains to be studied whether these observations are cell-type specific or cell-type independent.

Aggarwal and co-workers also reported that zerumbone can enhance anti-cancer effects of tumor necrosis factor-related apoptosis-inducing ligand (TRAIL) with up-regulation of TRAIL death receptors DR5 and DR4 in a variety of cancer cells [50]. They also observed that zerumbone down-regulated the expression of anti-apoptotic protein c-FLIP in HCT116 cells thus causing enhancement of TRAIL-induced apoptosis. However, they found that other anti-apoptotic proteins such as XIAP, survivin, Bcl-2 and Bcl- $\mathrm{X}_{\mathrm{L}}$ were not significantly affected by zerumbone treatment in HCT116 cells. As TRAIL-induced apoptosis is known to be regulated by reactive oxygen species (ROS), Aggarwal and co-workers investigated the effect of ROS scavengers N-acetylcysteine (NAC) and glutathione (GSH) on the zerumbone-induced up-regulation of death receptor (DR) 4 and 5. The researchers observed that pretreatment of HCT116 cells with these ROS scavengers reduced the zerumbone-induced up-regulation of DR4 and DR5 in a dosedependent manner. Moreover, the NAC pre-treatment has been found to attenuate several effects of zerumbone treatment such as TRAIL-induced cell death enhancement, increase in activation of caspase-3, caspase-8 and poly (ADP-ribose) polymerase (PARP)cleavage; all pointing to the potential role of ROS in the induction of TRAIL death receptors by zerumbone. The upregulation of DR4 and DR5 by zerumbone was also shown to be mediated through the activation of MAPK pathway. For instance, a dose-dependent suppression of the up-regulation of DR4 and DR5 by zerumbone treatment was observed in HCT116 cells after the treatment of extracellular signal-regulated kinase $1 / 2$ (ERK1/2) inhibitor
PD98059. Consistent with these observations, zerumbone treatment has been shown to cause time-dependent activation of ERK1/ 2 proteins as evident from time-dependent up-regulation of the corresponding p-ERK1/2 proteins. The effect of the sesquiterpene on the ERK1/2 activation was also shown to be dependent on ROS as the antioxidant NAC caused inhibition of this activation. They also observed that silencing of ERK1 in HCT116 cells with siRNA against ERK1 caused suppression in zerumbone-mediated upregulation of ERK1. These studies thus indicate that zerumbone enhanced TRAIL-induced apoptosis though the ROS-mediated activation of ERK1/2 through the up-regulation of DR4 and DR5. Fig. 2 summarizes the effect of zerumbone on NF-кB-, RANKL-, and TRAIL-induced signaling cascades in tumor cells.

Aggarwal and co-workers also reported the potential suppressive effects of zerumbone on the chemokine receptor CXCR4 [51], which is found to be overexpressed in several tumors [52]. Zerumbone suppressed both human epidermal growth factor receptor 2 (HER2)-induced CXCR4 expression in MCF7 breast cancer cell lines and constitutive CXCR4 expression in a variety of cancer cell lines such as leukemia (KBM-5), myeloma (U266), head and neck squamous cell carcinomas (SCC4), kidney carcinoma (A293), lung adenocarcinoma (H1299) and pancreatic carcinoma (PANC-1, PANC-28 and MIA PaCa-2). Interestingly, zerumbone did not suppress CXCR4 through proteasomal and lysosomal degradation. Moreover, reverse transcription polymerase chain reaction (RT-PCR) assay revealed time-dependent suppression of CXCR4 mRNA expression upon zerumbone treatment, thus indicating its ability to modulate CXCR4 expression at transcriptional level. It has previously been shown that NF- $\kappa$ B activation regulates HER2 overexpression in breast cancer [53]. Furthermore, using chromatin immunoprecipitation assay with an anti-p65 antibody and CXCR4 primer, they found that zerumbone suppressed NF- $\mathrm{B}$

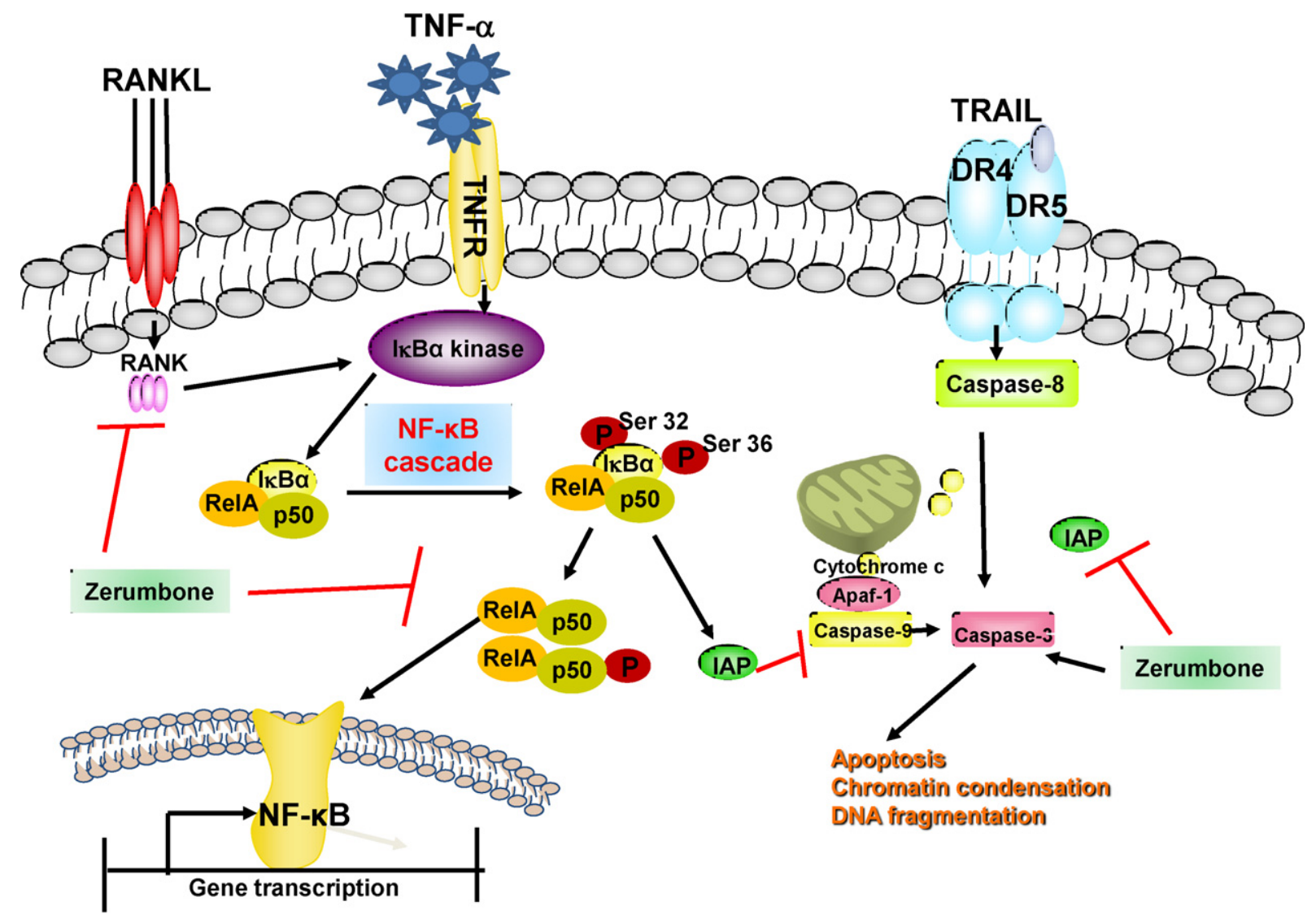

Fig. 2. Effect of zerumbone on NF-кB-, RANKL-, and TRAIL-induced signaling cascades in tumor cells. 
binding to CXCR4 promoter region and also inhibited CXCL12induced invasion of breast and pancreatic cancer cells.

Several studies demonstrate the role of phase II xenobiotic metabolizing enzymes in the cellular detoxification against the insult of oxidative, electrophilic, genotoxic and carcinogenic chemicals [54,55]. Nakamura and co-workers reported doseand time-dependent induction of GSTP1, a pi-class glutathione Stransferase that is known to cause detoxification of many pollutants and carcinogens, by zerumbone treatment in rat normal liver epithelial cell line (RL34 cell line) [56]. Structural analogs of zerumbone namely, 8 -hydroxy- $\alpha$-humulene and $\alpha$-humulene, both lacking the electrophilic carbonyl functionality of the parent compound, were found to be inactive in these assays, indicating the critical role of the $\alpha, \beta$-unsaturated carbonyl group in zerumbone for its biological activity. The researchers also reported that zerumbone not only stabilized the nuclear factor (erythroidderived 2)-like 2 (Nrf2) protein, a transcription factor that is known to be involved in the activation of gene expression of several phase II enzymes including glutathione S-transferase P (GSTP1) from proteasomal degradation, but also induced its nuclear translocation. Furthermore, they observed that zerumbone potentiated the mRNA expression of Nrf2-dependent enzyme genes GSTP1, $\mathrm{NAD}(\mathrm{P}) \mathrm{H}$ quinone oxidoreductase 1 (NQO1) and hemeoxygenase-1 (HO-1), indicating its potential role as an activator of the Nrf2/ARE-dependent detoxification pathway. Zerumbone treatment, although led to an initial lowering of cellular GSH level, was reported to cause significant elevation in the intracellular GSH level over a period of time. Zerumbone also potentiated mRNA expression of GSH-biosynthesis-related genes, suggesting its critical role in enhancing the GSH biosynthesis at the transcriptional level. Time-dependent suppressive effect of zerumbone on $\mathrm{H}_{2} \mathrm{O}_{2} / \mathrm{Fe}^{2+}$ induced lipid peroxidation was also observed, thereby further supporting its role as a potent antioxidant. In line with these findings, Murakami and co-workers recently reported their studies on the ability of zerumbone to induce mRNA expression of phase II drug metabolizing enzymes GSTP1 and NQO1 in RAW264.7 mouse macrophages [57].

The exact molecular mechanisms by which zerumbone exerts its effect on Nrf2 is not known yet. However, Nakamura and coworkers proposed the idea of an electrophilic attack on the Nrf2 repressor protein Kelch-like ECH-associated protein 1 (Keap1) as likelihood. In the Nrf2-Keap1 protein complex, the Keap1 interacts with Nrf2 and this interaction facilitates the degradation of the later by $26 \mathrm{~S}$ proteasome. Thus if zerumbone, because of the presence of its electrophilic functionality could interact with Keap1 (which has 25 cysteine residues with at least four of them having strongly nucleophilic sulfhydryl groups), such interaction(s) could potentially liberate the Nrf2 from Keap1 and this might account for the observed increased stability and nuclear localization of Nrf2 in zerumbone treated cell lines. Although this remains purely hypothetical, some recent data support this hypothesis. For instance, using zerumbone bound Sepharose gel, Murakami and co-workers performed in vitro pull-down assays from cell lysate preparation of mouse macrophages (RAW264.7 cells) and their assays identified Keap1, homologous to human Rantigen (HuR) and $\beta$-actin as in vitro co-valent targets of this phytochemical [57]. A nucleophilic attack of an amino acid residue (presumably a reactive cysteine residue) from these target protein(s) to the olefin in the $\alpha, \beta$ unsaturated carbonyl group of zerumbone (thus forming the co-valent bond) was proposed as a plausible molecular mechanism for the binding. The observation of HuR as an in vitro target is also interesting as along with several other proteins in the adenosine and uridine rich element-binding proteins (ARE-BPs) the HuR is known to play regulatory roles in the COX-2 mRNA stabilization [58,59]. The in vitro pull-down assays, although may not exactly represent the actual in vivo binding specificities; but may shed some light on the potential molecular targets of zerumbone.

Recently, Lee and co-workers reported that zerumbone caused cross-linking of heat shock protein (HSP) 27 with inhibition of monomeric form of the protein in the human lung adenocarcinoma cells NCI-H1299 [60]. Overexpression of HSPs has been observed in several cancer cells and members in this family of proteins have been suspected as one of the causative agents for resistance of cancer cells against radiation treatment and chemotherapeutic agents $[61,62]$. They found that zerumbone was inserted between disulfide linkages in the HSP27 dimers and this cross-linking altered the normal dimerization of the protein. The $\alpha, \beta-$ unsaturated carbonyl group in zerumbone was found to be essential for this cross-linking as the structural analogs of the phytochemical namely $\alpha$-humulene and 8-hydroxy humulene, both lacking the $\alpha, \beta$-unsaturated carbonyl functionality, failed to cause the cross-linking. Furthermore, zerumbone pre-treatment before $\gamma$-ray irradiation exposure was found to inhibit the binding affinity of HSP27 with apoptotic molecules such as PKC $\delta$ and cytochrome $\mathrm{c}$ and it induced sensitization of the cells to radiation treatment. Zerumbone also induced radiosensitization in $\mathrm{NCI}-$ H1299 xenograft mice, validating the strategy under the in vivo setting. Identification of HSP27 as a molecular target and the reported effect of altered oligomerization by zerumbone illustrate its potential to circumvent HSP27-mediated resistance of cancer cells.

Many chronic inflammatory disorders including cancer and arthritis often involve excessive bone resorption. The process is mediated by osteoclast cells which are generated from hematopoietic cells by the action of several entities including the receptor activator of NF- $\mathrm{B}$ (RANK) and its ligand RANKL [63]. As the latter is a member in the TNF family of cytokines, agents that can suppress its signaling have been suggested to have therapeutic potential against inflammatory disorders. Aggarwal and co-workers reported that zerumbone can inhibit RANKL-induced NF- $\kappa B$ activation in mouse macrophages (RAW264.7 cells) in a dose- and time-dependent manner [63]. The compound inhibited RANKLinduced $\mathrm{I} \kappa \mathrm{B} \alpha$ phosphorylation and degradation, IKK activation and osteoclastogenesis. They also observed that zerumbone was able to inhibit human breast cancer and multiple myeloma cells-induced osteoclast differentiation. Recent findings from our lab also indicate that zerumbone can exert its anticancer effects in renal cell carcinoma cell lines through the suppression of signal transducer and activator of transcription 3 /janus kinase 2 signaling cascades (unpublished data). These promising in vitro results point to the need of more pre-clinical studies to assess the therapeutic potential of this sesquiterpene.

\section{In vivo anti-cancer effects of zerumbone}

Zerumbone has been reported to inhibit tumor growth in various animal models of inflammation and cancer (Table 2 ). In the first published in vivo study with zerumbone, Tanaka and coworkers reported that oral administration of the sesquiterpene suppressed azoxymethane-induced colonic aberrant crypt foci in male F344 rats in a dose-dependent manner [64]. Subsequently, Murakami and co-workers reported that oral administration of zerumbone caused suppression of dextran sodium sulfate-induced acute colitis in female ICR mice [65]. The researchers also observed that zerumbone lowered the levels of inflammatory biomarkers IL$1 \beta$, TNF- $\alpha$ and prostaglandin E2 in the mouse colonic mucosa. In a subsequent paper Murakami and co-workers reported suppressive effect of zerumbone on 7,12-dimethylbenz[ $\alpha$ ]anthracene- and TPA-induced initiation and promotion of skin tumors in ICR mice [66]. Topical application of zerumbone was found to enhance mRNA expression levels of anti-oxidative and phase II xenobiotic 
Table 2

Selected in vivo anti-inflammatory and anti-cancer effects of zerumbone.

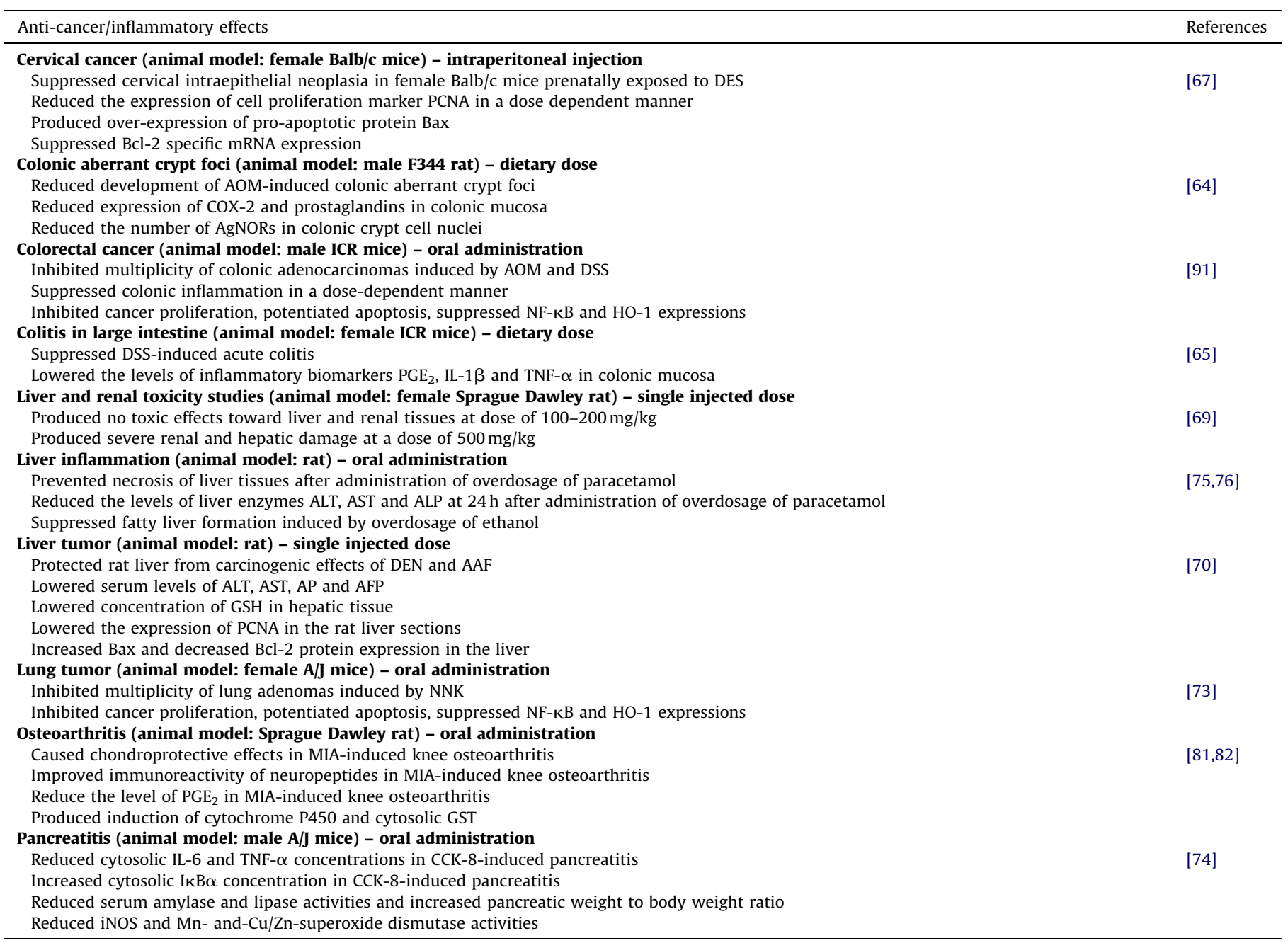

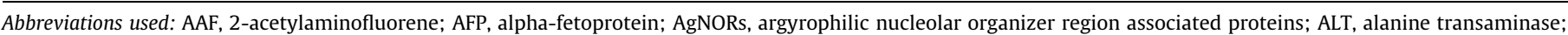

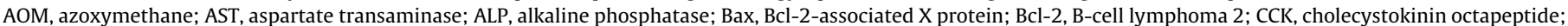

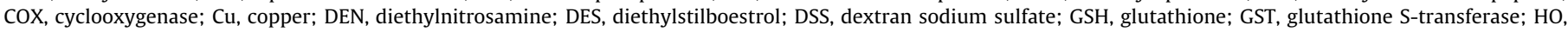

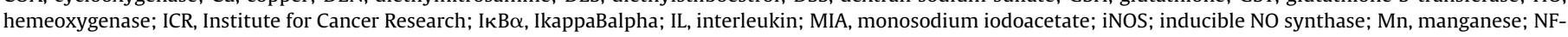

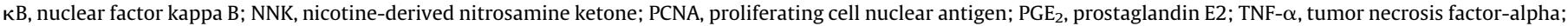
$\mathrm{Zn}$, zinc.

metabolizing enzymes manganese superoxide dismutase (MnSOD), glutathione peroxidise-1 (GPX-1), GST-P1 and NQO1 in the mouse epidermis. These results are in agreement with the in vitro studies of Nakamura and co-workers discussed earlier in this review. Another important observation included suppression of TPA-induced COX-2 expression and phosphorylation of ERK1/2 in the mouse skin. Furthermore, zerumbone was found to suppress TPA-induced leukocyte maturation and dermal infiltration as well as activation stages of skin tumors in the mice. These studies clearly indicate the therapeutic potential of zerumbone in the prevention and treatment of skin tumors at both initiation and promotion stages.

Zerumbone has been shown to suppress cervical intraepithelial neoplasia in female Balb/c mice, which were prenatally exposed to diethylstilbestrol (DES), at efficacy close to that of the anti-cancer drug cisplatin [67]. Dose-dependent reduction in the expression of the cell proliferation marker proliferating cell nuclear antigen (PCNA), over-expression of the pro-apoptotic protein Bax and suppression of the Bcl-2 specific mRNA expression are other important effects reported to be caused by zerumbone treatment in this model. The comparable efficacy of zerumbone with that of cisplatin shows great potential for alternative treatment for cervical cancer. This is intriguing considering serious side-effects of the latter. Published studies so far indicate that zerumbone exerts little cytotoxic and genotoxic effects at doses used for most experimental studies. For instance, a dose as high as $2000 \mathrm{mg} / \mathrm{kg}$ b.w. was determined to be the lethal dose in rats after intraperitoneal administration of the sesquiterpene [68]. Similarly, single injected doses of zerumbone at $100-200 \mathrm{mg} / \mathrm{kg}$ produced no toxic effects toward liver and renal tissues of female Sprague Dawley rats [69].

Anti-tumorigenic effects of zerumbone were also reported on diethylnitrosamine (DEN) (single injected dose) and dietary 2acetylaminoflurene (AAF)-induced liver tumors in rats [70]. The sesquiterpene was reported to protect rat liver from carcinogenic effects of DEN and AAF. Serum levels of alanine transaminase (ALT), aspartate transaminase (AST), alkaline phosphatase (AP) and alpha-fetoprotein (AFP) were all reported to be lowered by zerumbone administration. Zerumbone also caused lowering of glutathione concentration in hepatic tissues and PCNA expression in rat liver sections. Furthermore, in line with the previous findings on the effect of the sesquiterpene on Bax and Bcl-2 proteins, Abdul 
and co-workers observed that zerumbone can induce increase in Bax protein and decrease in $\mathrm{Bcl}-2$ protein expression in the rat liver.

Both colorectal and lung cancers are known to have chronic inflammation as a high risk factor for carcinogenesis [71,72]. As zerumbone has been suggested to modulate many inflammationrelated molecular targets, it has also been investigated as a chemopreventive agent against these cancers. Oral administration of zerumbone at 100,250 and $500 \mathrm{ppm}$ was reported to significantly inhibit multiplicity of colonic adenocarcinoma in male ICR mice that were previously induced with colon cancer using single injected doses of azoxymethane with oral doses of colitis-inducing agent dextran sodium sulfate [73]. Zerumbone was also found to significantly suppress colonic inflammation in a dose-dependent manner. Similarly, oral administration of zerumbone at 250 and $500 \mathrm{ppm}$ was reported to significantly inhibit multiplicity of lung adenomas in female $\mathrm{A} / \mathrm{J}$ mice with $4-(\mathrm{N}-$ methyl-N-nitrosamino)-1-(3-pyridyl)-1-butanone-induced lung tumors [73]. In both cases, feeding of zerumbone resulted in inhibition of cancer proliferation, apoptosis and suppression of NF$\kappa \mathrm{B}$ and HO-1 proteins. Anti-inflammatory effects of zerumbone were further evident from the reported ability of the compound to reduce cytosolic IL- 6 and TNF- $\alpha$ and increase I $\mathrm{B} \alpha \alpha$ concentration in cholecystokinin octapeptide (CCK-8)-induced experimental pancreatitis in male Wistar rats [74]. Zerumbone also reduced serum amylase and lipase activities and pancreatic weight to body weight ratio in the treated specimens. Furthermore, zerumbone treatment led to reduction in iNOS and $\mathrm{Mn}$ - and $\mathrm{Cu} / \mathrm{Zn}$-superoxide dismutase activities, thereby indicating its anti-oxidant potential in mice models.

There are several other examples of anti-inflammatory effects of zerumbone in animal models. For instance, zerumbone was reported to reduce overdosage of paracetamol-induced inflammation of liver tissues in male Sprague-Dawley rats [75]. Zerumbone was also found to prevent necrosis of the rat liver tissues. Zerumbone pretreatment also reduced the levels of rat liver enzymes ALT, AST and ALP at $24 \mathrm{~h}$ after administration of overdosage of paracetamol. This sesquiterpene was also found to suppress fatty liver formation due to overdosage of ethanol administration in rats, further supporting the hepatoprotective property [76]. Intraperitoneal administration of zerumbone at a dose of $5,10,50$ and $100 \mathrm{mg} / \mathrm{kg}$ was reported to produce dosedependent inhibition of carrageenan-induced paw edema in mice, indicating anti-inflammatory activity [77]. Zerumbone also suppressed granulomatous tissue formation in cotton pelletinduced granuloma test in mice [77]. In a recent study, zerumbone was reported to protect mouse cornea from ultraviolet B (UVB)induced inflammatory photokeratitis with inhibition of NF- $\kappa$, iNOS and TNF- $\alpha$ expression, abrogation of nuclear translocation of $\mathrm{NF}-\kappa \mathrm{B}$, reduction of malonyldialdehyde accumulation and increase of GSH and glutathione reductase levels [78]. Dietary zerumbone was also observed to protect mice cornea from UVB-induced cataractogenesis [79].

Many pro-inflammatory chemical mediators and pro-inflammatory cytokines are known to play critical roles in the pathogenesis of osteoarthritis [80]. Zerumbone has been investigated for potential curative effects in chemical-induced osteoarthritis in animal models. For instance, oral administration of zerumbone at a dose of $2 \mathrm{~mL} / \mathrm{kg}$ for a period of 4 weeks produced chondroprotective effects in monosodium iodoacetate (MIA)induced knee osteoarthritis in Sprague Dawley rats [81]. Zerumbone was also found to improve immunoreactivity of neuropeptides in MIA-induced knee osteoarthritis models [82]. This might have implications in modifying inflammatory environments typically established at the joints during osteoarthritis. Oral administration of zerumbone was reported to reduce the level of $\mathrm{PGE}_{2}$ in rats with MIA-induced knee osteoarthritis, indicating its potential anti-inflammatory activity in these models. Also, as zerumbone is known to prevent COX-2 expression in articular tissues, it has been suggested that the compound may indirectly suppress inflammatory cytokines production and hence provide another plausible mechanism of its beneficial effects in osteoarthritis models. Besides its useful effects in osteoarthritis models, zerumbone was also found suppress osteolytic bone metastases in a dose-dependent manner in human breast cancer-bearing athymic nude mice, pointing to its potential as a therapeutic agent for osteoporosis and cancer-induced bone loss [63].

\section{Conclusion}

In summary, this minireview clearly describes that zerumbone can affect multiple cell-signaling pathways involved in inflammation and cancer and thus has therapeutic potential against various inflammatory diseases and cancer. Both in vitro and in vivo data from published literature so far suggest that zerumbone can modulate multiple molecular targets that play pivotal role in both chronic inflammation and carcinogenesis. However, in future more detailed investigations are needed to completely understand its mechanisms of actions and chemopreventive/therapeutic potential against different cancers. Also, whether zerumbone can modulate identical multiple pro-inflammatory signaling cascades in distinct tumors should also be evaluated. Interestingly, zerumbone has been found to be bioavailable following oral administration in rats and mice, although no data are available for its pharmacokinetic and pharmacodynamic profiles in humans. Hence, detailed toxicological and clinical studies are needed to fully realize the therapeutic potential of this wonderful drug provided by Mother Nature.

\section{Conflict of interest}

\section{None.}

\section{Acknowledgments}

This work was supported by grants from National Kidney Foundation [R-184-000-196-592] and Academic Research Fund [R184-000-207-112] to GS. This work was also supported by the Korea Science and Engineering Foundation (KOSEF) grant funded by the Korean Ministry of Education, Science and Technology (MoEST) (No. 2011-0006220) to KSA. APK was supported by grants from the National Medical Research Council of Singapore [R-713-000-124-213] and Cancer Science Institute of Singapore, Experimental Therapeutics I Program [R-713-001-011-271].

\section{References}

[1] Ichikawa H, Nakamura Y, Kashiwada Y, Aggarwal BB. Anticancer drugs designed by mother nature: ancient drugs but modern targets. Curr Pharm Des 2007;13:3400-16.

[2] Butler MS, Newman DJ. Mother Nature's gifts to diseases of man: the impact of natural products on anti-infective, anticholestemics and anticancer drug discovery. Prog Drug Res 2008;65(1):3-44.

[3] Gullett NP, Ruhul Amin AR, Bayraktar S, Pezzuto JM, Shin DM, Khuri FR, et al Cancer prevention with natural compounds. Semin Oncol 2010;37:258-81.

[4] Newman DJ, Cragg GM. Natural products as sources of new drugs over the 30 years from 1981 to 2010. J Nat Prod 2012;75:311-35.

[5] Deorukhkar A, Krishnan S, Sethi G, Aggarwal BB. Back to basics: how natural products can provide the basis for new therapeutics. Expert Opin Investig Drugs 2007; 16:1753-73.

[6] Aggarwal BB, Sethi G, Baladandayuthapani V, Krishnan S, Shishodia S. Targeting cell signaling pathways for drug discovery: an old lock needs a new key. J Cell Biochem 2007:102:580-92.

[7] Bolzani Vda S, Davies-Coleman M, Newman DJ, Singh SB, Gordon M, Cragg GM. A man for all natural products. J Nat Prod 2012;75:309-10. 
[8] Harvey AL. Natural products in drug discovery. Drug Discov Today 2008;13:894-901.

[9] Aggarwal BB, Van Kuiken ME, Iyer LH, Harikumar KB, Sung B. Molecular targets of nutraceuticals derived from dietary spices: potential role in suppression of inflammation and tumorigenesis. Exp Biol Med (Maywood) 2009;234:825-49.

[10] Shanmugam MK, Kannaiyan R, Sethi G. Targeting cell signaling and apoptotic pathways by dietary agents: role in the prevention and treatment of cancer. Nutr Cancer 2011;63:161-73.

[11] Oldham RK, Dillman RO. Monoclonal antibodies in cancer therapy: 25 years of progress. J Clin Oncol 2008;26:1774-7.

[12] Butler MS. Natural products to drugs: natural product-derived compounds in clinical trials. Nat Prod Rep 2008;25:475-516.

[13] Macarron R, Banks MN, Bojanic D, Burns DJ, Cirovic DA, Garyantes T, et al. Impact of high-throughput screening in biomedical research. Nat Rev Drug Discov 2011;10:188-95.

[14] Cragg GM, Grothaus PG, Newman DJ. Impact of natural products on developing new anti-cancer agents. Chem Rev 2009;109:3012-43.

[15] Khosla C, Keasling JD. Metabolic engineering for drug discovery and development. Nat Rev Drug Discov 2003;2:1019-25.

[16] Mukherji S, van Oudenaarden A. Synthetic biology: understanding biological design from synthetic circuits. Nat Rev Genet 2009;10:859-71.

[17] Newman D, Cragg G. Natural products in medicinal chemistry. Bioorg Med Chem 2009;17:2120.

[18] Shu L, Cheung KL, Khor TO, Chen C, Kong AN. Phytochemicals: cancer chemoprevention and suppression of tumor onset and metastasis. Cancer Metastasis Rev 2010;29:483-502.

[19] Lee KW, Bode AM, Dong Z. Molecular targets of phytochemicals for cancer prevention. Nat Rev Cancer 2011;11:211-8

[20] Surh YJ. Xenohormesis mechanisms underlying chemopreventive effects of some dietary phytochemicals. Ann N Y Acad Sci 2011;1229:1-6.

[21] Aggarwal BB, Shishodia S, Sandur SK, Pandey MK, Sethi G. Inflammation and cancer: how hot is the link? Biochem Pharmacol 2006;72:1605-21.

[22] Surh YJ. Cancer chemoprevention with dietary phytochemicals. Nat Rev Cancer 2003;3:768-80.

[23] Tan AC, Konczak I, Sze DM, Ramzan I. Molecular pathways for cancer chemoprevention by dietary phytochemicals. Nutr Cancer 2011;63:495-505.

[24] Aggarwal BB, Shishodia S. Molecular targets of dietary agents for prevention and therapy of cancer. Biochem Pharmacol 2006;71:1397-421.

[25] Gupta SC, Kim JH, Kannappan R, Reuter S, Dougherty PM, Aggarwal BB. Role of nuclear factor kappaB-mediated inflammatory pathways in cancer-related symptoms and their regulation by nutritional agents. Exp Biol Med (Maywood) $2011 ; 236: 658-71$.

[26] Surh YJ, Kundu JK, Na HK, Lee JS. Redox-sensitive transcription factors as prime targets for chemoprevention with anti-inflammatory and antioxidative phytochemicals. J Nutr 2005;135:2993S-3001S

[27] Kundu JK, Surh YJ. Breaking the relay in deregulated cellular signal transduction as a rationale for chemoprevention with anti-inflammatory phytochemicals. Mutat Res 2005;591:123-46.

[28] Aggarwal BB, Vijayalekshmi RV, Sung B. Targeting inflammatory pathways for prevention and therapy of cancer: short-term friend, long-term foe. Clin Cancer Res 2009;15:425-30.

[29] Reuter S, Gupta SC, Chaturvedi MM, Aggarwal BB. Oxidative stress, inflammation, and cancer: how are they linked? Free Radic Biol Med 2010;49:1603-16.

[30] Sethi G, Shanmugam MK, Ramachandran L, Kumar AP, Tergaonkar V. Multifaceted link between cancer and inflammation. Biosci Rep 2012;32:1-15.

[31] Aggarwal BB, Gehlot P. Inflammation and cancer: how friendly is the relationship for cancer patients? Curr Opin Pharmacol 2009;9:351-69.

[32] Aggarwal BB. Inflammation, a silent killer in cancer is not so silent! Curr Opin Pharmacol 2009;9:347-50.

[33] Kuraishy A, Karin M, Grivennikov SI. Tumor promotion via injury- and deathinduced inflammation. Immunity 2011;35:467-77.

[34] Maimone TJ, Baran PS. Modern synthetic efforts toward biologically active terpenes. Nat Chem Biol 2007;3:396-407.

[35] Shanmugam MK, Nguyen AH, Kumar AP, Tan BK, Sethi G. Targeted inhibition of tumor proliferation, survival, and metastasis by pentacyclic triterpenoids: potential role in prevention and therapy of cancer. Cancer Lett 2012;320:158-70.

[36] Yadav VR, Prasad S, Sung B, Kannappan R, Aggarwal BB. Targeting inflammatory pathways by triterpenoids for prevention and treatment of cancer. Toxins (Basel) 2010;2:2428-66.

[37] Sauerwein M, Becker H. Growth, terpenoid production and antibacterial activity of an in vitro culture of the liverwort Fossombronia pusilla. Planta Med 1990;56:364-7.

[38] Niedermeyer TH, Lindequist U, Mentel R, Gordes D, Schmidt E, Thurow K, et al. Antiviral terpenoid constituents of Ganoderma pfeifferi. J Nat Prod 2005;68:1728-31.

[39] Dhingra V, Vishweshwar Rao K, Lakshmi Narasu M. Current status of artemisinin and its derivatives as antimalarial drugs. Life Sci 2000;66:279-300.

[40] Ajikumar PK, Tyo K, Carlsen S, Mucha O, Phon TH, Stephanopoulos G. Terpenoids: opportunities for biosynthesis of natural product drugs using engineered microorganisms. Mol Pharm 2008;5:167-90.

[41] Fort DM, Ubillas RP, Mendez CD, Jolad SD, Inman WD, Carney JR, et al. Novel antihyperglycemic terpenoid-quinones from Pycnanthus angolensis. J Org Chem 2000;65:6534-9.

[42] Salminen A, Lehtonen M, Suuronen T, Kaarniranta K, Huuskonen J. Terpenoids: natural inhibitors of NF-kappaB signaling with anti-inflammatory and anticancer potential. Cell Mol Life Sci 2008;65:2979-99.
[43] Murakami A, Takahashi D, Kinoshita T, Koshimizu K, Kim HW, Yoshihiro A, et al. Zerumbone, a Southeast Asian ginger sesquiterpene, markedly suppresses free radical generation, proinflammatory protein production, and cancer cell proliferation accompanied by apoptosis: the alpha,beta-unsaturated carbonyl group is a prerequisite. Carcinogenesis 2002;23:795-802.

[44] Murakami A, Shigemori T, Ohigashi H. Zingiberaceous and citrus constituents, $1^{\prime}$-acetoxychavicol acetate, zerumbone, auraptene, and nobiletin, suppress lipopolysaccharide-induced cyclooxygenase-2 expression in RAW264.7 murine macrophages through different modes of action. J Nutr 2005;135:2987S92S.

[45] Takada Y, Murakami A, Aggarwal BB. Zerumbone abolishes NF-kappaB and IkappaBalpha kinase activation leading to suppression of antiapoptotic and metastatic gene expression, upregulation of apoptosis, and downregulation of invasion. Oncogene 2005;24:6957-69.

[46] Sethi G, Tergaonkar V. Potential pharmacological control of the NF-kappaB pathway. Trends Pharmacol Sci 2009;30:313-21.

[47] Li F, Sethi G. Targeting transcription factor NF-kappaB to overcome chemoresistance and radioresistance in cancer therapy. Biochim Biophys Acta 2010;1805:167-80.

[48] Shen HM, Tergaonkar V. NFkappaB signaling in carcinogenesis and as a potential molecular target for cancer therapy. Apoptosis 2009;14:348-63.

[49] Sakinah SA, Handayani ST, Hawariah LP. Zerumbone induced apoptosis in liver cancer cells via modulation of Bax/Bcl-2 ratio. Cancer Cell Int 2007:7:4.

[50] Yodkeeree S, Sung B, Limtrakul P, Aggarwal BB. Zerumbone enhances TRAILinduced apoptosis through the induction of death receptors in human colon cancer cells: evidence for an essential role of reactive oxygen species. Cancer Res 2009;69:6581-9.

[51] Sung B, Jhurani S, Ahn KS, Mastuo Y, Yi T, Guha S, et al. Zerumbone downregulates chemokine receptor CXCR4 expression leading to inhibition of CXCL12-induced invasion of breast and pancreatic tumor cells. Cancer Res 2008;68:8938-44

[52] Muller A, Homey B, Soto H, Ge N, Catron D, Buchanan ME, et al. Involvement of chemokine receptors in breast cancer metastasis. Nature 2001;410:50-6.

[53] Biswas DK, Iglehart JD. Linkage between EGFR family receptors and nuclear factor kappaB (NF-kappaB) signaling in breast cancer. J Cell Physiol 2006;209:645-52.

[54] Hayes JD, Ellis EM, Neal GE, Harrison DJ, Manson MM. Cellular response to cancer chemopreventive agents: contribution of the antioxidant responsive element to the adaptive response to oxidative and chemical stress. Biochem Soc Symp 1999;64:141-68.

[55] Talalay P. Chemoprotection against cancer by induction of phase 2 enzymes. Biofactors 2000;12:5-11.

[56] Nakamura Y, Yoshida C, Murakami A, Ohigashi H, Osawa T, Uchida K. Zerumbone, a tropical ginger sesquiterpene, activates phase II drug metabolizing enzymes. FEBS Lett 2004;572:245-50.

[57] Ohnishi K, Irie K, Murakami A. In vitro covalent binding proteins of zerumbone, a chemopreventive food factor. Biosci Biotechnol Biochem 2009;73:1905-7.

[58] Dixon DA, Tolley ND, King PH, Nabors LB, McIntyre TM, Zimmerman GA, et al Altered expression of the mRNA stability factor HuR promotes cyclooxygenase-2 expression in colon cancer cells. J Clin Invest 2001;108:1657-65.

[59] Serini S, Fasano E, Piccioni E, Monego G Cittadini AR, Celleno L, et al. DHA induces apoptosis and differentiation in human melanoma cells in vitro: involvement of HuR-mediated COX-2 mRNA stabilization and beta-catenin nuclear translocation. Carcinogenesis 2012;33:164-73.

[60] Choi SH, Lee YJ, Seo WD, Lee HJ, Nam JW, Kim J, et al. Altered cross-linking of HSP27 by zerumbone as a novel strategy for overcoming HSP27-mediated radioresistance. Int J Radiat Oncol Biol Phys 2011;79:1196-205.

[61] Ciocca DR, Calderwood SK. Heat shock proteins in cancer: diagnostic, prognostic, predictive, and treatment implications. Cell Stress Chaperones 2005; 10:86-103

[62] Calderwood SK, Khaleque MA, Sawyer DB, Ciocca DR. Heat shock proteins in cancer: chaperones of tumorigenesis. Trends Biochem Sci 2006;31:164-72.

[63] Sung B, Murakami A, Oyajobi BO, Aggarwal BB. Zerumbone abolishes RANKLinduced NF-kappaB activation, inhibits osteoclastogenesis, and suppresses human breast cancer-induced bone loss in athymic nude mice. Cancer Res 2009;69:1477-84

[64] Tanaka T, Shimizu M, Kohno H, Yoshitani S, Tsukio Y, Murakami A, et al. Chemoprevention of azoxymethane-induced rat aberrant crypt foci by dietary zerumbone isolated from Zingiber zerumbet. Life Sci 2001;69: 1935-45.

[65] Murakami A, Hayashi R, Tanaka T, Kwon KH, Ohigashi H, Safitri R. Suppression of dextran sodium sulfate-induced colitis in mice by zerumbone, a subtropical ginger sesquiterpene, and nimesulide: separately and in combination. Biochem Pharmacol 2003;66:1253-61.

[66] Murakami A, Tanaka T, Lee JY, Surh YJ, Kim HW, Kawabata K, et al. Zerumbone, a sesquiterpene in subtropical ginger, suppresses skin tumor initiation and promotion stages in ICR mice. Int J Cancer 2004;110:481-90.

[67] Abdelwahab SI, Abdul AB, Devi N, Taha MM, Al-zubairi AS, Mohan S, et al. Regression of cervical intraepithelial neoplasia by zerumbone in female Balb/c mice prenatally exposed to diethylstilboestrol: involvement of mitochondriaregulated apoptosis. Exp Toxicol Pathol 2010;62:461-9.

[68] Al-Zubairi AS, Abdul AB, Syam MM. Evaluation of the genotoxicity of zerumbone in cultured human peripheral blood lymphocytes. Toxicol In Vitro 2010;24:707-12

[69] Ibrahim MY, Abdul ABH, Ibrahim TAT, Abdelwahab SI, Elhassan MM, Syam MM. Evaluation of acute toxicity and the effect of single injected doses of 
zerumbone on the kidney and liver functions in Sprague Dawley rats. Afr J Biotechnol 2010;9:4442-50.

[70] Taha MM, Abdul AB, Abdullah R, Ibrahim TA, Abdelwahab SI, Mohan S. Potential chemoprevention of diethylnitrosamine-initiated and 2-acetylaminofluorene-promoted hepatocarcinogenesis by zerumbone from the rhizomes of the subtropical ginger (Zingiber zerumbet). Chem Biol Interact 2010;186: 295-305.

[71] Azad N, Rojanasakul Y, Vallyathan V. Inflammation and lung cancer: roles of reactive oxygen/nitrogen species. J Toxicol Environ Health B Crit Rev 2008; $11: 1-15$.

[72] Xie J, Itzkowitz SH. Cancer in inflammatory bowel disease. World J Gastroenterol 2008;14:378-89.

[73] Kim M, Miyamoto S, Yasui Y, Oyama T, Murakami A, Tanaka T. Zerumbone, a tropical ginger sesquiterpene, inhibits colon and lung carcinogenesis in mice. Int J Cancer 2009;124:264-71.

[74] Szabolcs A, Tiszlavicz L, Kaszaki J, Posa A, Berko A, Varga IS, et al. Zerumbone exerts a beneficial effect on inflammatory parameters of cholecystokinin octapeptide-induced experimental pancreatitis but fails to improve histology. Pancreas 2007;35:249-55.

[75] Fakurazi SHI, Lip JM, Shanthi G, Nanthini U, Shamima AR. Hepatoprotective action of zerumbone against paracetamol induced hepatotoxicity. J Med Sci 2009;9:161-4

[76] Fakurazi S, Lip HI, Shanthi JMG. The effect of pretreatment of zerumbone on fatty liver following ethanol induced hepatotoxicity. J Biol Sci 2008;8:1348-51.

[77] Sulaiman MR, Perimal EK, Akhtar MN, Mohamad AS, Khalid MH, Tasrip NA, et al. Anti-inflammatory effect of zerumbone on acute and chronic inflammation models in mice. Fitoterapia 2010;81:855-8.

[78] Chen BY, Lin DP, Wu CY, Teng MC, Sun CY, Tsai YT, et al. Dietary zerumbone prevents mouse cornea from UVB-induced photokeratitis through inhibition of NF-kappaB, iNOS, and TNF-alpha expression and reduction of MDA accumulation. Mol Vis 2011;17:854-63.

[79] Chen BY, Lin DP, Su KC, Chen YL, Wu CY, Teng MC, et al. Dietary zerumbone prevents against ultraviolet B-induced cataractogenesis in the mouse. Mol Vis 2011;17:723-30.

[80] Kapoor M, Martel-Pelletier J, Lajeunesse D, Pelletier JP, Fahmi H. Role of proinflammatory cytokines in the pathophysiology of osteoarthritis. Nat Rev Rheumatol 2011;7:33-42.
[81] Al-Saffar FJ, Ganabadi S, Fakurazi S, Yaakub H, Lip M. Chondroprotective effects of zerumbone on monosodium iodoacetate induced osteoarthritis in rats. J Appl Sci 2010;10:248-60. http://dx.doi.org/10.3923/jas.2010.248.260.

[82] Al-Saffar FJ, Ganabadi S, Fakurazi S, Yaakub H. Zerumbone improved immunoreactivity of neuropeptides in monosodium iodoacetate induced knee osteoarthritis in rat. Afr J Biotechnol 2011;10:3646-53. http://dx.doi.org/ 10.5897/AJB10.2371.

[83] Abdelwahab SI, Abdul AB, Mohan S, Taha MM, Syam S, Ibrahim MY, et al. Zerumbone induces apoptosis in T-acute lymphoblastic leukemia cells. Leuk Res 2011;35:268-71.

[84] Abdul AB, Abdelwahab SI, Bin Jalinas J, Al-Zubairi AS, Taha MM. Combination of zerumbone and cisplatin to treat cervical intraepithelial neoplasia in female BALB/c mice. Int J Gynecol Cancer 2009;19:1004-10.

[85] Keong YS, Alitheen NB, Mustafa S, Abdul Aziz S, Abdul Rahman M, Ali AM. Immunomodulatory effects of zerumbone isolated from roots of Zingiber zerumbet. Pak J Pharm Sci 2010;23:75-82.

[86] Sciullo EM, Vogel CF, Wu D, Murakami A, Ohigashi H, Matsumura F. Effects of selected food phytochemicals in reducing the toxic actions of TCDD and p, $\mathrm{p}^{\prime}-$ DDT in U937 macrophages. Arch Toxicol 2010;84:957-66.

[87] Xian M, Ito K, Nakazato T, Shimizu T, Chen CK, Yamato K, et al. Zerumbone, a bioactive sesquiterpene, induces G2/M cell cycle arrest and apoptosis in leukemia cells via a Fas- and mitochondria-mediated pathway. Cancer Sci 2007;98:118-26.

[88] Huang GC, Chien TY, Chen LG, Wang CC. Antitumor effects of zerumbone from Zingiber zerumbet in P-388D1 cells in vitro and in vivo. Planta Med 2005;71:219-24.

[89] Murakami A, Takahashi M, Jiwajinda S, Koshimizu K, Ohigashi H. Identification of zerumbone in Zingiber zerumbet Smith as a potent inhibitor of 12-Otetradecanoylphorbol-13-acetate-induced Epstein-Barr virus activation. Biosci Biotechnol Biochem 1999;63:1811-2.

[90] Eguchi A, Kaneko Y, Murakami A, Ohigashi H. Zerumbone suppresses phorbol ester-induced expression of multiple scavenger receptor genes in THP-1 human monocytic cells. Biosci Biotechnol Biochem 2007;71: 935-45.

[91] Murakami A, Miyamoto M, Ohigashi H. Zerumbone, an anti-inflammatory phytochemical, induces expression of proinflammatory cytokine genes in human colon adenocarcinoma cell lines. Biofactors 2004;21:95-101. 